\title{
BMJ Open Protocol for the development of Core Outcome Sets for Early intervention trials to Prevent Obesity in CHildren (COS-EPOCH)
}

\author{
Vicki Brown (D , ,,2 Marj Moodie, ${ }^{1,2}$ Huong Ngoc Quynh Tran, ${ }^{1,2}$ Marufa Sultana, ${ }^{1}$ \\ Kylie Elizabeth Hunter (D) ,2,3 Rebecca Byrne, ${ }^{2,4}$ Dorota Zarnowiecki (D) ,2,5 \\ Anna Lene Seidler (D) ,2,3 Rebecca Golley, ${ }^{2,5}$ Rachael Taylor, ${ }^{2,6}$ Kylie D Hesketh, ${ }^{2,7}$ \\ Karen Matvienko-Sikar (ib) 8
}

To cite: Brown V, Moodie M, Tran HNQ, et al. Protocol for the development of Core Outcome Sets for Early intervention trials to Prevent Obesity in CHildren (COS-EPOCH). BMJ Open 2021;11:e048104. doi:10.1136/ bmjopen-2020-048104

- Prepublication history for this paper is available online. To view these files, please visit the journal online (http://dx.doi. org/10.1136/bmjopen-2020048104).

Received 17 December 2020 Accepted 21 June 2021

Check for updates

(C) Author(s) (or their employer(s)) 2021. Re-use permitted under CC BY-NC. No commercial re-use. See rights and permissions. Published by BMJ.

For numbered affiliations see end of article.

Correspondence to

Dr Vicki Brown;

victoria.brown@deakin.edu.au

\section{ABSTRACT}

Introduction Childhood overweight and obesity is prevalent in the first 5 years of life, and can result in significant health and economic consequences over the lifetime. The outcomes currently measured and reported in randomised controlled trials of early childhood obesity prevention interventions to reduce this burden of obesity are heterogeneous, and measured in a variety of ways. This variability limits the comparability of findings between studies, and contributes to research waste. This protocol presents the methodology for the development of two core outcome sets (COS) for obesity prevention interventions in children aged from 1 to 5 years from a singular development process: (1) a COS for interventions targeting physical activity and sedentary behaviour and (2) a COS for interventions targeting child feeding and dietary intake. Core outcomes related to physical activity and sedentary behaviour in children aged $\leq 1$ year will also be identified to complement an existing COS for early feeding interventions, and provide a broader set of core outcomes in this age range. This will result in a suite of $\mathrm{COS}$ useful for measuring and reporting outcomes in early childhood obesity prevention studies, including multicomponent interventions.

Methods and analysis Development of the COS will follow international best practice guidelines. A scoping review of trial registries will identify commonly reported outcomes and associated measurement instruments. Key stakeholders involved in obesity prevention, including policy-makers/ funders, parents, researchers, health practitioners and community and organisational stakeholders will participate in an e-Delphi study and consensus meeting regarding inclusion of outcomes in the COS. Finally, recommended outcome measure instruments will be identified through literature review and group consensus.

Ethics and dissemination Deakin University Human Research Ethics Committee (HEAG-H 231_2020). The COS will be disseminated through peer-reviewed publications and engagement with key stakeholders.

\section{INTRODUCTION}

Overweight and obesity in early childhood is a significant issue, with 41 million children

\section{Strengths and limitations of this study}

- Development of core outcome sets (COS) will assist in determining the outcomes that should be measured, and how they should be measured, following Core Outcome Measures in Effectiveness Trials guidelines.

- Engagement with key stakeholders and a steering group comprising experts in the field of early childhood obesity prevention will ensure relevance and facilitate dissemination and uptake of the COS.

- The large number of possible outcomes for inclusion may present a risk for lack of consensus on core outcomes or outcome measurement instruments.

- This risk will be minimised through the development of a suite of COS, through a singular COS development process.

aged from birth to 5 years now affected globally. ${ }^{1}$ Obesity is a significant risk factor for several chronic conditions generally occurring both in childhood and later in life, and the associated economic burden is high. ${ }^{2}$ Addressing childhood overweight and obesity has been identified as critically important, ${ }^{1}$ particularly given that children with overweight and obesity are five times more likely to be obese in adulthood compared with their healthy weight peers. ${ }^{3}$ Altering or maintaining obesity trajectories into adulthood should ideally commence before 6 years of age ${ }^{4}$ highlighting the need for effective and cost-effective childhood obesity prevention interventions in the early years of life.

There are a number of risk factors for early childhood overweight and obesity, including poor nutrition, insufficient physical activity or sleep and excess sedentary behaviours. ${ }^{1}$ Given this wide range of risk factors, there are currently a large number of outcomes 
reported from obesity prevention intervention studies in children aged from birth to 5 years. ${ }^{5-7}$ There are also a wide range of methods currently used for measuring relevant outcomes, which limits consistency and comparability of findings between studies and can lead to research waste. ${ }^{8}$ Variation also makes evidence synthesis via retrospective meta-analysis very difficult, if not impossible, due to limitations in combining data that has been collected, measured or reported using different methods. ${ }^{9}$

Core outcome sets (COS) are agreed minimum sets of outcomes recommended for measurement in studies of specific conditions or areas of health or healthcare. ${ }^{10}$ COS aim to improve the consistency of measurement and reporting of outcomes from studies, potentially leading to better informed resource allocation and decisionmaking through improved comparability and transparency of study findings. The development of COS using well-defined guidelines such as those proposed by the Core Outcome Measures in Effective Trials (COMET) initiative minimises the risk of increased burden on researchers. ${ }^{10}$ In addition, the benefits that standardisation of outcomes brings to the field in terms of identifying effective approaches far out-weighs any potential increase in research burden. While COS are the recommended minimum outcomes for use in studies it is acknowledged that in some instances not all outcomes can be evaluated and in this context a clear explanation for why a COS outcome was not used is sufficient. ${ }^{10}$ COS are currently in development for obesity prevention interventions in children delivered in the school setting and for children with obesity aged over 5 years exposed to physical activity interventions. ${ }^{11}$ A COS has been developed to identify the minimum outcomes that should be measured and reported in trials of early feeding interventions to prevent childhood obesity, ${ }^{12}{ }^{13}$ recommending 26 outcomes for inclusion in trials of feeding interventions involving children aged $\leq 1$ year of age.

To date, COS that could be applied more broadly to early childhood obesity prevention interventions spanning the wider range of risk factors, and for prevention interventions in children aged from birth to 5 years, are not available. This is despite the growing number of early childhood obesity prevention interventions targeting multiple risk factors. ${ }^{415}$ Early childhood represents a time of rapid growth and development, particularly in infancy (up to 1 year of age). Early childhood obesity prevention interventions typically take place within a broad range of settings (eg, community, home, early childhood education and care). Intervention component/s related to lifestyle also commonly target a number of risk factors (eg, diet, physical activity, sedentary behaviour, sleep, parent/ caregiver practices) at the individual (ie, child/parent/ caregiver) or family level.

To account for this heterogeneity, this paper describes the protocol for the development of a suite of COS for trials of early childhood obesity prevention interventions, developed through a singular COS process. We will build on a published COS for trials of early feeding interventions ${ }^{13}$ to develop a COS for obesity prevention interventions targeting the broader range of risk factors and commencing either prenatally or from birth until children are $\leq 1$ year of age. This will result in tailored advice on the outcomes recommended for collection and reporting in interventions targeting multiple risk factors in infancy. We will also develop two COS for obesity prevention interventions targeting the broader range of lifestyle-related risk factors and commencing in children aged from $>1$ to 5 years. The first COS will be useful for studies of physical activity, sleep and sedentary behaviour interventions. The second COS will be useful for studies of feeding and dietary interventions in children aged from $>1$ to 5 years. The development of the proposed suite of COS minimises the potential risk of achieving a lack of consensus given the large number of expected outcomes from such heterogeneous interventions. When considered holistically, the suite of COS produced will provide valuable information to trialists of interventions targeting multiple risk factors for obesity in the early years of life. Publication of this protocol aims to enhance transparency of this COS development process, and may also help to reduce potential bias. ${ }^{10}$

\section{Project oversight}

An international Steering Group will be formed to provide expert oversight and guide the development of the COS, chaired by the lead author (VB). The members of the steering group will be selected based on their expertise in early childhood obesity prevention intervention and outcome measurement. Initially members will be identified through the member and affiliate base of the National Health and Medical Research Council funded Centre for Research Excellence in the Early Prevention of Obesity in Childhood (APP1101675). These steering group members will then recommend international experts and key contacts within the field of early childhood obesity prevention intervention for invitation as Steering Group members.

\section{METHODS AND ANALYSIS}

This study was prospectively registered on the COMET Initiative registry of COS (registration number 1679, http://www.comet-initiative.org/Studies/Details/1679). The study will be conducted between June 2020 and June 2022. Development of the COS will follow the Core Outcome Set-STAndards for Development (COS-STAD) recommendations. ${ }^{10}{ }^{16}$ The reporting of this protocol follows the recommendations of the Core Outcome SetSTAndardised Protocol Items Statement. ${ }^{17}$

COS development generally involves defining 'what' to measure, and then deciding 'how' to measure these outcomes. ${ }^{10}$ A first step towards defining 'what' to measure might typically consist of a systematic review of outcomes being reported in relevant studies, or searches of clinical trial registries for reported outcomes from relevant randomised controlled trial (RCTs). ${ }^{10}$ Further steps 


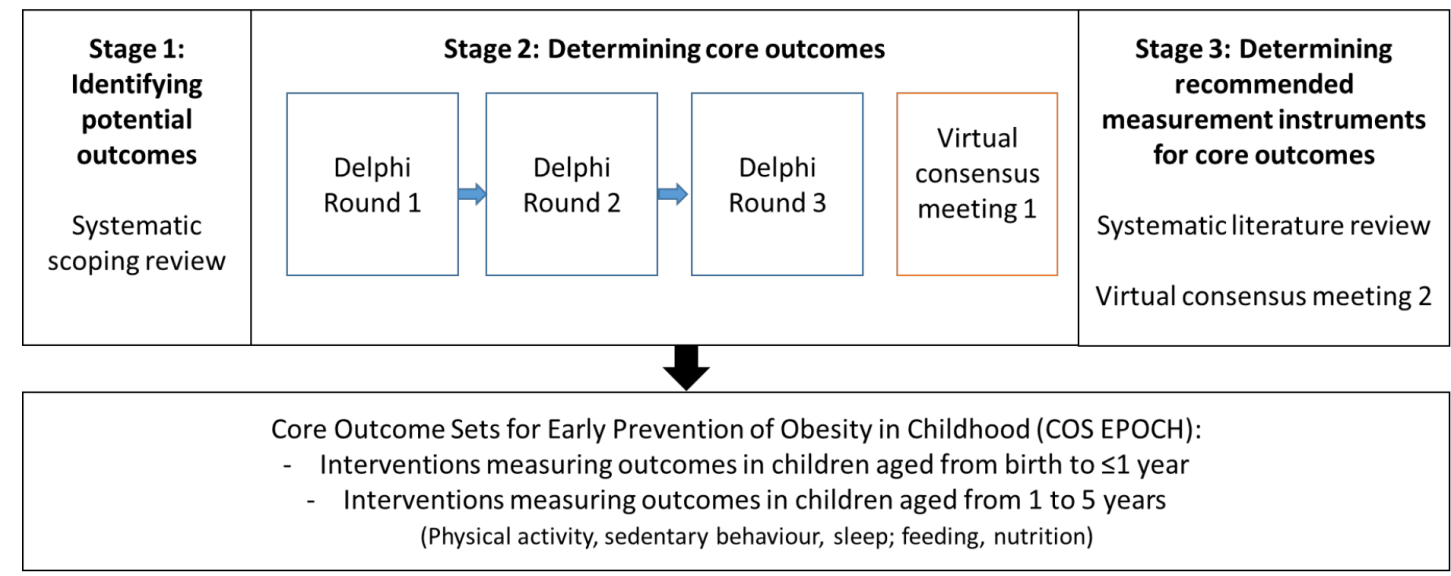

Figure 1 Overview of project stages.

in the development of a COS include achieving consensus agreement using methods such as expert panels, Delphi surveys and consensus meetings. ${ }^{1016}$ Development of this COS will therefore consist of three stages (figure 1):

Stage 1-A scoping review of early childhood obesity prevention intervention RCTs, identifying potential outcomes and outcome measurement instruments.

Stage 2-A modified Delphi study to determine core outcomes by relevant stakeholder group, followed by a consensus meeting to finalise core outcome recommendations.

Stage 3-Determination of recommended measurement instruments for core outcomes, through literature review and consensus meeting.

\section{Stage 1: identifying potential outcomes}

A systematic scoping review of early childhood obesity prevention intervention RCTs will be undertaken, to identify potential outcomes for inclusion in stage 2 of our study. Scoping reviews are useful for clarifying working definitions and conceptual boundaries of a topic or field ${ }^{18}$ and aim to provide an overview or map of the evidence in a particular area. ${ }^{19}$ The scoping review will follow Joanna Briggs Institute guidelines for conducting a scoping review. ${ }^{18}$ The scoping review protocol has been published on Open Science framework (https://osf.io/; osf.io/ snv5e) and reporting of the scoping review will follow the Preferred Reporting Items for Systematic Reviews and Meta-Analyses Extension for Scoping Reviews. ${ }^{20}$

We will conduct a search of publicly available clinical trial registries (clinicaltrials.gov and via the WHO International Clinical Trials Registry Platform), using a predefined search strategy (table 1) to identify the outcomes of interest in registered early childhood obesity prevention intervention RCTs.

Identified records will be exported into Microsoft Excel and screened for inclusion by two reviewers, with any conflicts resolved by a third reviewer. Registered studies that meet the following inclusion criteria will be included:

- Randomised.

- In any stage of research (eg, recruiting, active, complete).

- Have the aim of preventing childhood obesity (ie, stated as a primary or secondary aim; specified within the trial registry as condition/disease: obesity).

- Start interventions in the first 5 years of childhood, or antenatally.

- If interventions start antenatally, they must continue interventions for at least 6 months postnatally. This will allow us to include interventions that begin antenatally but with significant intervention content after the birth of the child. Not being prescriptive around duration for interventions that start postnatally allows for the broadest range of outcomes to be included within the scoping review, although the scoping review analysis will include intervention duration and measurement time.

- Undertake implementation of an intervention that includes a component related to lifestyle (eg, diet, parent/caregiver practices, physical activity, sedentary

\begin{tabular}{|c|c|}
\hline Registry & Search strategy \\
\hline $\begin{array}{l}\text { WHO International } \\
\text { Clinical Trials } \\
\text { Registry Platform } \\
\text { (WHO ICTRP) }\end{array}$ & $\begin{array}{l}\text { The "Advanced search" option will be } \\
\text { selected, and the following fields will be } \\
\text { populated: } \\
\text { Title: prevent OR prevention } \\
\text { Condition: obesity OR overweight } \\
\text { Recruitment status: all } \\
\text { Limit: search for clinical trials in children } \\
\text { Status: all }\end{array}$ \\
\hline Clinicaltrials.gov & $\begin{array}{l}\text { The "Advanced search" option will be } \\
\text { selected, and the following fields will be } \\
\text { populated: } \\
\text { Condition or disease: Obesity OR obese } \\
\text { OR adiposity OR overweight } \\
\text { Age: Child (Birth-17 years) } \\
\text { Type of studies: interventional studies } \\
\text { Other terms: prevent OR prevention }\end{array}$ \\
\hline
\end{tabular}


behaviour, sleep). Lifestyle interventions are defined as interventions that promote change in lifestyle behaviours for the prevention of unhealthy weight gain. $^{21}$

- Any length of follow-up time.

Studies will be excluded if they include a targeted or treatment intervention for overweight or obesity or for those at risk of overweight or obesity (ie, participant inclusion criteria above healthy weight for either parent or child; identify as treatment trial type in register; targeted to participants with specific body weight or body mass index percentile inclusion criteria that includes above healthy weight) or if they are undertaken in an admitted patient hospital setting or in special groups (eg, preterm children, children with cerebral palsy). Studies will also be excluded if they are undertaken in the primary school or after-school setting with primary school aged children, despite the fact that some children beginning school will be less than 5 years of age. This exclusion criteria was designed to avoid duplication with a COS for obesity prevention interventions delivered in the school setting that is currently under development. ${ }^{11}$ Studies will also be excluded if the unit of intervention does not include the child (ie, higher-level outcomes reported, not including child level health outcomes; intervention content only at the environmental level or intervention content delivered only to individuals within organisations (eg, healthcare professionals, childcare providers), with no parent/caregiver/child-directed content). This does not preclude interventions directed at parents/caregivers only, but with child outcomes.

Study inclusions will also be cross-referenced to the recently published Cochrane review study by Brown $e t a l^{22}$ that included obesity prevention intervention RCTs in children aged under 5 years. The Cochrane review search strategy $^{22}$ will be updated to November 2020 and rerun in Ovid Medline, and potential studies will be screened for inclusion by two reviewers. This will ensure our dataset reflects both studies that have been registered in trial registries, and studies that may not have been registered but reported results.

A data extraction tool will be developed in Microsoft Excel, based on COMET recommendations. ${ }^{10}$ Outcome extraction from the source will be verbatim by two independent reviewers, to maintain transparency. ${ }^{10}$ Data will include trial registration number, public or scientific study title, study acronym, study start date, study completion date, recruitment status, study aim and/or hypothesis, RCT study type, recruitment country, setting, intervention summary, comparator summary, participant inclusion criteria, sample size, participant age, primary and secondary outcomes reported, outcome measurement instruments, outcome definitions, time points of assessment, links to publications, primary study contact and sponsor information. Where links to relevant publications have been provided, we will search these publications for more detailed data. Where links to relevant publications are not supplied, we will search for unlinked publications using keyword searches related to the trial name and lead author in the Scopus and GoogleScholar databases. Any additional data from linked or unlinked publications will also be extracted verbatim, to maintain transparency ${ }^{10}$ and will be extracted by two reviewers.

It is expected that a long and varied list of outcomes and outcome measurement methods will be generated. ${ }^{12}$ While recently there has been more published research exploring obesity intervention taxonomies, ${ }^{23} 24$ there has been less focus on taxonomy structures focusing specifically on outcomes. ${ }^{25}$ To the best of our knowledge, a comprehensive and validated taxonomy fit for our specific purpose has not been developed. Therefore we will take a data-driven approach, whereby outcomes will be grouped into outcome domains based on relevant risk factor/s for obesity (eg, physical activity, sedentary behaviour, dietary intake, sleep, parent/caregiver practices) and applicable outcome domains from a taxonomy developed for outcomes in medical research at an individual-participant level (eg, anthropometry, emotional functioning/wellbeing, cognitive functioning, economic). ${ }^{25}$ Outcomes with similar definitions or themes within each domain will be merged, via a consensus process with members of the steering group with expertise in each outcome domain. ${ }^{12}$ Subdomains (eg, child feeding practices, screen time) will be identified based on key literature conceptualising outcome domains. ${ }^{26-29}$ Categorisation of each verbatim outcome definition to an outcome domain and sub-domain will be performed initially by one reviewer (VB), with final consensus sought from members of the steering group. Outcome frequencies will be estimated and presented in outcome matrices to visually represent the frequency, consistency, and disparity of outcome reporting across studies, ${ }^{12}$ stratified by age (ie, interventions in children aged $\leq 1$ year; and $>1$ to 5 years) and risk factor/s targeted (ie, nutrition, physical activity, sedentary behaviour, multiple risk factors). Outcome matrices will be based on the Outcome Reporting Bias in Trials project outcome matrix,${ }^{30}$ as recommended by the COMET initiative ${ }^{10}$ and used in a previous COS study investigating early feeding outcomes. ${ }^{12}$

The quality of included trials with respect to their measurement properties will not be assessed as part of stage 1 of this project, in accordance with some of the most recently published research on COS development. ${ }^{31} 32$ While previous studies have conducted quality assessment of the measurement properties of included studies by adapting six items from the Consensus-based Standards for the selection of health Measurement Instruments $($ COSMIN $),{ }^{33}$ these criteria have not been well-validated for this purpose ${ }^{31}$ and there is a lack of transparency in how scores can be attributed to studies with multiple outcomes that are reported heterogeneously. For instance, one of the criterion asks 'Is the primary outcome clearly defined so that another researcher would be able to reproduce its measurement? Where appropriate, this should include clear description of time points, the person measuring the outcome, how the outcome was measured (eg, tools and 
methods used) and where the outcome was measured.' It is not clear however how studies that report more than one primary outcome (perhaps even with differences in reporting clarity between multiple primary outcomes), or that may include a clear description of time points but not the person measuring the outcome, should be scored. Further, descriptors of reporting quality are not considered integral components of the review stage for COS development. ${ }^{31}$

\section{Stage 2: determining core outcomes}

An electronic Delphi (e-Delphi) study will be undertaken, in accordance with published recommendations on outcome consensus using the Delphi technique and the recommendations of the COMET Initiative. ${ }^{34}$ The Delphi technique is a widely used methodology in health research, ${ }^{35}$ with the approach taking the findings from the Stage 1 scoping review and aiming to achieve consensus on core outcomes for inclusion in the COS.

Stakeholders, including (1) policy-makers/funders, (2) parents/caregivers, (3) researchers, (4) clinicians and health practitioners (including representatives from professional organisations such as dietetic and paediatric associations) and (5) community and organisational stakeholders to obesity prevention interventions (eg, representatives from settings where interventions are undertaken such as Maternal Child Health centres, childcare; health promotion organisations), will be invited to participate. Published guidelines encourage the inclusion of a diverse range of relevant stakeholders in COS development, including health service users, policy-makers, experts and the public. ${ }^{10}$ A central component of the COMET methodology is the recognition that multiple stakeholders can provide expert insights and input in determining core outcomes. ${ }^{10}$ Parents in this instance are an excellent example where individuals not typically considered 'experts' can provide some of the most useful information because they are the end users of the interventions, and the ones who engage in feeding and physical activity behaviours. As such their contributions are essential. This is similarly applicable to other stakeholder groups who are either directly involved in research, policy or practice around childhood obesity, and child dietary and physical activity behaviours. An interesting finding of this component of the work will include whether there are differences in opinion between different stakeholder groups. ${ }^{34}$ As there is no consensus on the number of participants or rounds required for a Delphi study, ${ }^{36}$ membership to the e-Delphi panels will be balanced across stakeholder groups and capped at 150 participants to maintain feasibility (ie, a maximum of 30 participants per stakeholder panel). Recent evidence suggests that a smaller sample size of between 8 and 15 participants may be sufficient for relatively homogeneous participant groups, but that larger sample sizes can help to ensure generalisability. ${ }^{35}$

Recruitment of participants will be undertaken using purposive and snowball sampling. ${ }^{37}$ Preliminary lists of potential policy-maker/funder, researcher, clinician/ healthcare practitioner and community/organisational stakeholders will be generated from key contacts of steering group members and the obesity prevention literature. Information on the study and invitations to participate will be sent to publicly available email addresses. Those consenting to participate from these stakeholder groups will be encouraged to circulate study details among their professional networks. Potential parent/ caregiver participants will be recruited using social media and recruitment posters at sites that parents/caregivers likely visit (eg, childcare centres). Inclusion criteria for parents/caregivers will include having at least one child aged from birth to 5years; being fluent in English; and, being able to freely give informed consent. All consenting parent/caregiver participants will be asked to circulate study information to friends meeting the inclusion criteria.

Participants will be allocated a unique identifier to anonymise their responses, and will be asked to commit to completion of the 3 rounds of the e-Delphi study. Rounds will be open for a 3-week period, and to maximise response rates an automated reminder email will be sent to participants yet to complete their survey on days 7 and 14. If required, additional strategies such as extending survey deadline(s) and personalised reminders will be discussed with the Steering Group and may be used to boost response. The time between rounds will not exceed 4 weeks, which will allow for data analysis and setup but not be so long as to risk increased participant attrition over time.

The steering group will be consulted in the development of the online questionnaires, which will be developed using the COMET DelphiManager software and pilot-tested to ensure feasibility. Outcomes will be presented by COS and domain, with the ordering of domains randomised. Outcomes per domain will be presented in alphabetical order and a plain language definition of each outcome will be provided. Participants will rate the importance of each outcome based on a 9-point Likert scale anchored between 1 and 9, and will be asked to enter comments on their choice of ranking for each outcome. The scale for responses will be based on Grading of Recommendations Assessment, Development and Evaluation (GRADE), where 1-3 signifies an outcome that is 'not that important', 4-6 'important but not critical' and 7-9 'critically important'. ${ }^{38}$ Participants will also be asked to list up to three additional outcomes they feel should be included in the survey. Responses will be collected and analysed both within and between groups of panel members (group mean, median, strength of agreement using mean absolute deviation from the median (MADM). ${ }^{39}$ Levels of agreement using the MADM will be defined using values from the literature (low >1.41; moderate 1.08-1.41; high $<1.08$ ) ${ }^{39}$ Consensus will be defined as ${ }^{4041}$ :

1. Consensus include as a core outcome: over $75 \%$ of participants in each stakeholder group score the outcome domain as 'critically important' for inclusion in 
the relevant COS AND $<15 \%$ of participants in each stakeholder group score outcome domain 'of limited importance'.

2. Consensus do not include as a core outcome: over $75 \%$ of participants in each stakeholder group score domain 'of limited importance' for inclusion in the relevant COS AND $<15 \%$ of participants in each stakeholder group score outcome domain 'critical'.

3. No consensus: all other combinations.

Additional outcomes listed by study participants in round 1 will be reviewed by the steering group for inclusion into round 2. During the second round of the survey, participants will receive a summary of their responses for round 1 and the distribution of scores by stakeholder group. Participants will be invited to review their round 1 ratings, and rerate outcomes from 1 to 9. Outcome ratings for round 2 will be analysed as for round 1 . The outcomes that have reached consensus for inclusion, and the outcomes where no consensus has been reached, will be included in round 3. Outcomes that reach consensus to not include as a core outcome will not be brought forward to round 3 . In round 3 participants will again receive a summary of their responses and the distribution scores by stakeholder group for these outcomes and will be asked to rerate for the final time. Ratings from round 3 will be analysed as per the previous two rounds to determine consensus on outcomes for inclusion, outcomes not to include and outcomes for which there is no consensus on inclusion or exclusion. Potential bias arising from participant attrition between rounds will be assessed by examining the differences in median round 1 scores of individual outcomes among those who do and do not complete later rounds. ${ }^{10}$

Results from the e-Delphi study will be narratively and quantitatively compared between stakeholder panels, and will be presented at a consensus meeting with key stakeholders and the Steering Group. Participants from the wider e-Delphi cohort will be asked at the end of the third round of the e-Delphi survey to express their interest in participating in two half-day consensus meetings over a 6-month period. If consent to participate is high, up to four members from each stakeholder group will be randomly selected to participate $(n=20)$. At a minimum, one member from each stakeholder group will be recruited to participate. The first consensus meeting will use the modified nominal group technique and will be conducted virtually in accordance with COVID-19 travel restrictions and to maximise input from international stakeholder experts.

The aim of this consensus meeting will be to develop the final suite of COS for early childhood obesity prevention interventions. At the start of the meeting, the study background, aims and a lay definition of a COS will be presented. The same process will then be followed to reach consensus on each COS. Participants will be presented with the outcomes for which consensus for inclusion and consensus to not include has been reached through the e-Delphi process, and will be asked to briefly discuss. The outcomes that have not reached consensus through the e-Delphi process will then be presented to participants. Participants will be asked to consider which outcomes they most and least strongly supported for inclusion. Following this discussion, participants will be asked to anonymously vote each outcome as 'yes' or 'no' for inclusion in the final COS. Outcomes $\geq 70 \%$ or more of participants rated as 'yes' for inclusion will be briefly discussed a final time, followed by a discussion on all other outcomes. Participants will be invited to discuss the order of importance of outcomes, the similarity of outcomes (both within and between the COS), the relative importance of outcomes and the feasibility of collecting and reporting each outcome. Following this discussion, a final voting process will be undertaken. For inclusion in the final COS, $\geq 70 \%$ of participants will be required to vote 'yes' for inclusion of an outcome. Findings of the full COS process will be reported following the Core Outcome Set-Standards for Reporting guidelines. ${ }^{42}$

\section{Stage 3: determining recommended measurement for core outcomes}

It is also important to establish how the outcomes in a COS should be defined and measured. ${ }^{10}$ We will follow the recommendations of the joint initiative between COMET and COSMIN for selected outcome measurement instruments for outcomes included in a COS. ${ }^{43}$ Outcome measurement instruments commonly utilised in early childhood obesity prevention interventions will be identified across the studies in our scoping review (stage 1) and frequency of outcome measurement instruments will be reported. A systematic literature review will be conducted in PubMed, MedLine and Embase by two reviewers, aiming to identify and critically appraise, compare and summarise the quality of the measurement properties of the identified outcome measurement instruments for early childhood obesity prevention interventions identified in the scoping review. ${ }^{44}$ Validated search filters for finding studies on measurement properties are available from the COSMIN website, and will be used. ${ }^{45} 46$

Studies will be included in the systematic literature review if the outcome measurement instrument measures the construct of interest in children aged from birth to 5 years, including by either self-report or parent/caregiver report. Included studies should aim to summarise the development of the outcome measurement instrument, or to evaluate one or more of its measurement properties or its interpretability. ${ }^{44}$ The COSMIN Risk of Bias checklist will be used to assess the methodological quality of the measurement properties of outcome measurement instruments. The quality of evidence and strength of recommendations will align with the COSMIN and GRADE guidelines. ${ }^{47}$

Outcome measurement instruments will be ranked for inclusion in the relevant COS according to the quality of evidence for all measurement properties and presented to key stakeholders and the steering group at the second virtual consensus meeting. Outcome measurement 
instruments will be presented according to published criteria for good measurement properties, ${ }^{43}$ alongside the minimum requirements for inclusion of an instrument in a COS (ie, at least high-quality evidence for good content validity and for good internal consistency; and if the outcome measurement instrument is feasible). ${ }^{43}$ After group discussion participants will be asked to anonymously vote on outcome measurement instruments for inclusion into the COS. Outcome measurement instruments that $\geq 70 \%$ or more of participants rated as 'yes' for inclusion will be discussed. Where possible, only one outcome measurement instrument will be selected for each outcome in the relevant COS, following the final round of anonymous voting by meeting participants.

\section{ETHICS AND DISSEMINATION}

Ethics approval has been obtained from the Deakin University Human Research Ethics Committee (DUHREC; HEAG-H 231_2020). A dissemination plan for the COS for Early Prevention of Obesity in CHildhood (COS-EPOCH) will be developed by the steering group early in the project. The involvement of experts and key stakeholders in the design of the COS will facilitate uptake. ${ }^{10} \mathrm{~A}$ two-page infographic summary of each COS will also be developed, and will be sent to all study participants and stakeholders for early childhood obesity prevention intervention. Study findings will be reported in peer-reviewed publications and presented on relevant websites (such as the National Collaborative on Childhood Obesity Research and the World Obesity Federation) and at international conferences.

COS are increasingly recognised as valuable research tools, and have been actively endorsed by trialists, research funding bodies, regulatory authorities, systematic review groups including the Cochrane Collaboration and journal editors. ${ }^{11}$ This study aims to develop a suite of COS for early childhood obesity prevention interventions, through a singular COS development process. Together, these COS will provide trialists with agreed, standardised sets of outcomes spanning the early childhood time frame that takes into account this unique period of child development and incorporates interventions targeting multiple risk factors. The multiplicity of potential outcomes for inclusion into any COS will be a significant challenge in development. By following rigorous methodological processes and involving a number of key stakeholder groups, we hope to minimise this challenge and provide guidance to the growing number of researchers conducting trials in this important field. The COS will also recommend outcome measurement instruments for data collection that can contribute to improved evidence synthesis across early childhood obesity prevention intervention studies in the future.

\section{Author affiliations}

${ }^{1}$ Deakin Health Economics, Institute for Health Transformation, Global Obesity Centre (GLOBE), School of Health and Social Development, Deakin University,

Geelong, Victoria, Australia

${ }^{2}$ Centre for Research Excellence in the Early Prevention of Obesity in Childhood, University of Sydney, Sydney, New South Wales, Australia

${ }^{3}$ NHMRC Clinical Trials Centre, The University of Sydney, Sydney, New South Wales, Australia

${ }^{4}$ School of Exercise and Nutrition Sciences, Queensland University of Technology, Brisbane, Queensland, Australia

${ }^{5}$ Caring Futures Institute, College of Nursing and Health Sciences, Flinders University, Adelaide, South Australia, Australia

${ }^{6}$ Department of Medicine, University of Otago, Dunedin, New Zealand

${ }^{7}$ Institute for Physical Activity and Nutrition, Deakin University, Geelong, Victoria, Australia

${ }^{8}$ School of Public Health, University College Cork, Cork, Ireland

Twitter Vicki Brown @Vicki_BBB and Anna Lene Seidler @LeneSeidler

Contributors VB conceived the study, with significant input from all authors. VB, MS, MT, KEH, RB, DZ, ALS and KM-S will be involved in data acquisition, analysis and interpretation of data and will provide technical and administrative support. RG $\mathrm{RT}, \mathrm{KDH}, \mathrm{KM}-\mathrm{S}$ and MM will be involved in data analysis and interpretation, and will provide expert oversight of all aspects of the project. VB drafted the manuscript. All authors critically revised the manuscript and provided expert analysis. All authors have read and approved the manuscript.

Funding VB is supported by an Alfred Deakin Postdoctoral Research Fellowship. VB, MT, KEH, RB, DZ and ALS are researchers with the Centre for Research Excellence in the Early Prevention of Obesity in Childhood (CRE EPOCH, APPID1101675). KM-S receives support from a Health Research Board Applying Research into Policy and Practice Award (HRB-ARPP-A-2018-011).

Competing interests None declared.

Patient and public involvement Patients and/or the public were involved in the design, or conduct, or reporting, or dissemination plans of this research. Refer to the Methods section for further details.

Patient consent for publication Not required.

Provenance and peer review Not commissioned; externally peer reviewed.

Open access This is an open access article distributed in accordance with the Creative Commons Attribution Non Commercial (CC BY-NC 4.0) license, which permits others to distribute, remix, adapt, build upon this work non-commercially, and license their derivative works on different terms, provided the original work is properly cited, appropriate credit is given, any changes made indicated, and the use is non-commercial. See: http://creativecommons.org/licenses/by-nc/4.0/.

ORCID iDs

Vicki Brown http://orcid.org/0000-0003-2891-9476

Kylie Elizabeth Hunter http://orcid.org/0000-0002-2796-9220

Dorota Zarnowiecki http://orcid.org/0000-0003-0874-7830

Anna Lene Seidler http://orcid.org/0000-0002-0027-1623

Karen Matvienko-Sikar http://orcid.org/0000-0003-2777-6581

\section{REFERENCES}

1 World Health Organisation. Report of the commission on ending childhood obesity. Geneva: WHO, 2016.

2 Tremmel M, Gerdtham U-G, Nilsson PM, et al. Economic burden of obesity: a systematic literature review. Int J Environ Res Public Health 2017;14:435.

3 Simmonds M, Llewellyn A, Owen CG, et al. Predicting adult obesity from childhood obesity: a systematic review and meta-analysis. Obes Rev 2016;17:95-107.

4 Buscot M-J, Thomson RJ, Juonala M, et al. BMI trajectories associated with resolution of elevated youth $\mathrm{BMI}$ and incident adult obesity. Pediatrics 2018;141:e20172003.

5 Redsell SA, Edmonds B, Swift JA, et al. Systematic review of randomised controlled trials of interventions that AIM to reduce the risk, either directly or indirectly, of overweight and obesity in infancy and early childhood. Matern Child Nutr 2016;12:24-38.

6 Reilly JJ, Hughes AR, Gillespie J, et al. Physical activity interventions in early life aimed at reducing later risk of obesity and related non- 
communicable diseases: a rapid review of systematic reviews. Obes Rev 2019;20(Suppl 1):61-73.

7 Kerr JA, Loughman A, Knox A, et al. Nutrition-related interventions targeting childhood overweight and obesity: a narrative review. Obes Rev 2019;20(Suppl 1):45-60.

8 Webbe J, Brunton G, Ali S, et al. Developing, implementing and disseminating a core outcome set for neonatal medicine. BMJ Paediatr Open 2017;1:e000048.

9 Seidler AL, Hunter KE, Cheyne S, et al. A guide to prospective metaanalysis. BMJ 2019;367:I5342.

10 Williamson PR, Altman DG, Bagley H, et al. The COMET Handbook: version 1.0. Trials 2017;18:280.

11 Comet initiative. Core outcome measures in effectiveness trials (comet) initiative, 2020. Available: http://www.comet-initiative.org [Accessed 10 Sep 2020].

12 Matvienko-Sikar K, Griffin C, McGrath N, et al. Developing a core outcome set for childhood obesity prevention: a systematic review. Matern Child Nutr 2019;15:e12680.

13 Matvienko-Sikar K, Griffin C, Kelly C, et al. A core outcome set for trials of infant-feeding interventions to prevent childhood obesity. Int J Obes 2020;44:2035-43.

14 Seidler AL, Hunter KE, Johnson BJ, et al. Understanding, comparing and learning from the four epoch early childhood obesity prevention interventions: a multi-methods study. Pediatr Obes 2020;15:e12679.

15 Scott-Sheldon LAJ, Hedges LV, Cyr C, et al. Childhood obesity evidence base project: a systematic review and meta-analysis of a new taxonomy of intervention components to improve weight status in children 2-5 years of age, 2005-2019. Childhood Obesity 2020;16:S2-21-48.

16 Kirkham JJ, Davis K, Altman DG, et al. Core outcome Set-STAndards for development: the cos-stad recommendations. PLoS Med 2017;14:e1002447.

17 Kirkham JJ, Gorst S, Altman DG, et al. Core outcome setstandardised protocol items: the COS-STAP statement. Trials 2019;20:116.

18 Peters MDJ, Godfrey C, Mclnerney P. Chapter 11: Scoping reviews (2020 version). In: Joanna Briggs Institute Reviewer's Manual. Australia: JBI, 2020.

19 Munn Z, Peters MDJ, Stern C, et al. Systematic review or scoping review? guidance for authors when choosing between a systematic or scoping review approach. BMC Med Res Methodol 2018;18:143.

20 Tricco AC, Lillie E, Zarin W, et al. PRISMA extension for scoping reviews (PRISMA-ScR): checklist and explanation. Ann Intern Med 2018;169:467-73.

21 Hebden L, Chey T, Allman-Farinelli M. Lifestyle intervention for preventing weight gain in young adults: a systematic review and meta-analysis of RCTs. Obes Rev 2012;13:692-710.

22 Brown T, Moore THM, Hooper L, et al. Interventions for preventing obesity in children. Cochrane Database Syst Rev 2019;14.

23 Belle SH, Stevens J, Cella D, et al. Overview of the obesity intervention taxonomy and pooled analysis Working group. Trans Behav Med 2016;6:244-59.

24 King H, Magnus M, Hedges LV, et al. Childhood obesity evidence base project: methods for taxonomy development for application in taxonomic meta-analysis. Child Obes 2020;16:S2-7-S2-20.

25 Dodd S, Clarke M, Becker L, et al. A taxonomy has been developed for outcomes in medical research to help improve knowledge discovery. J Clin Epidemiol 2018;96:84-92.

26 Vaughn AE, Ward DS, Fisher JO, et al. Fundamental constructs in food parenting practices: a content map to guide future research. Nutr Rev 2016;74:98-117.

27 Mâsse LC, O'Connor TM, Tu AW, et al. Conceptualizing physical activity parenting practices using expert informed concept mapping analysis. BMC Public Health 2017;17:574.
28 Tremblay MS, Chaput J-P, Adamo KB, et al. Canadian 24-hour movement guidelines for the early years (0-4 years): an integration of physical activity, sedentary behaviour, and sleep. BMC Public Health 2017:17:874.

29 Okely AD, Ghersi D, Hesketh KD, et al. A collaborative approach to adopting/adapting guidelines - the Australian 24-hour movement guidelines for the early years (birth to 5 years): An integration of physical activity, sedentary behavior, and sleep. BMC Public Health 2017;17:869.

30 Kirkham JJ, Dwan KM, Altman DG, et al. The impact of outcome reporting bias in randomised controlled trials on a cohort of systematic reviews. BMJ 2010;340:c365.

31 D'Souza R, Hall C, Sermer M, et al. Development of a core outcome set for studies on cardiac disease in pregnancy (COSCarP): a study protocol. Trials 2020;21:300.

32 D'Souza R, Villani L, Hall C, et al. Core outcome set for studies on pregnant women with vasa previa (COVasP): a study protocol. BMJ Open 2020;10:e034018.

33 Mokkink LB, Terwee CB, Knol DL, et al. The COSMIN checklist for evaluating the methodological quality of studies on measurement properties: a clarification of its content. BMC Med Res Methodol 2010;10:22.

34 Sinha IP, Smyth RL, Williamson PR. Using the Delphi technique to determine which outcomes to measure in clinical trials: recommendations for the future based on a systematic review of existing studies. PLoS Med 2011;8:e1000393.

35 Trevelyan EG, Robinson PN. Delphi methodology in health research: how to do it? Eur J Integr Med 2015;7:423-8.

36 Diamond IR, Grant RC, Feldman BM, et al. Defining consensus: a systematic review recommends methodologic criteria for reporting of Delphi studies. J Clin Epidemiol 2014;67:401-9.

37 Keeney S, Hasson F, McKenna HP. The Delphi technique in nursing and health research. Wiley-Blackwell, 2011.

38 Williamson PR, Altman DG, Blazeby JM, et al. Developing core outcome sets for clinical trials: issues to consider. Trials 2012;13:132.

39 Taylor RM, Feltbower RG, Aslam N, et al. Modified international e-Delphi survey to define healthcare professional competencies for working with teenagers and young adults with cancer. BMJ Open 2016;6:e011361.

40 Hirsch M, Duffy JMN, Barker C, et al. Protocol for developing, disseminating and implementing a core outcome set for endometriosis. BMJ Open 2016;6:e013998.

41 De Meyer D, Kottner J, Beele H, et al. Delphi procedure in core outcome set development: rating scale and consensus criteria determined outcome selection. J Clin Epidemiol 2019;111:23-31.

42 Kirkham JJ, Gorst S, Altman DG, et al. Core outcome setstandards for reporting: the cos-star statement. PLoS Med 2016;13:e1002148.

43 Prinsen CAC, Vohra S, Rose MR. Guideline for selecting outcome measurement instruments for outcomes included in a core outcome set. The Netherlands: COMET and COSMIN, 2016.

44 Prinsen CAC, Mokkink LB, Bouter LM, et al. COSMIN guideline for systematic reviews of patient-reported outcome measures. Qual Life Res 2018;27:1147-57.

45 Terwee CB, Jansma EP, Riphagen II, et al. Development of a methodological PubMed search filter for finding studies on measurement properties of measurement instruments. Qual Life Res 2009;18:1115-23.

46 COSMIN. Consensus-based standards for the selection of health Measurment instruments (COSMIN), 2020. Available: https://www. cosmin.nl/ [Accessed 14 Sep 2020].

47 GRADE working group. Grade, 2020. Available: https://www. gradeworkinggroup.org/ [Accessed 14 Sep 2020]. 